\title{
SOME INTRINSIC COORDINATES ON TEICHMÜLLER SPACE
}

\author{
CLIFFORD J. EARLE ${ }^{1}$
}

\begin{abstract}
We give a new construction of intrinsic global coordinates on the Teichmüller space $T_{p}$ of closed Riemann surfaces of genus $p>2$. Our construction produces an injective holomorphic map from $T_{p}$ into the space of Schottky groups of genus $p$.
\end{abstract}

1. Introduction. Since the Teichmüller space $T_{p}$ of closed Riemann surfaces of genus $p \geqslant 2$ is a complex analytic manifold of dimension $n=3 p-3$, any injective holomorphic map $f: T_{p} \rightarrow C^{n}$ defines a set of global coordinate functions on $T_{p}$. We call these coordinates intrinsic if the coordinates $f(t)$ are determined from the marked Riemann surface $t$ alone and do not depend on the choice of a basepoint $t_{0}$ in $T_{p}$. In this paper we describe a new way to define intrinsic coordinates on $T_{p}$.

We should emphasize that we are defining complex coordinates for the complex manifold $T_{p}$. The problem of finding real analytic coordinates was solved classically with the help of Fuchsian groups. The first global complex coordinates were found by Bers [2], using quasi-fuchsian groups. The Bers coordinates depend on the choice of a basepoint. Maskit [5] defined the first intrinsic (complex) coordinates. Our coordinates are closer in spirit to the Bers coordinates since we use quasifuchsian groups. It would, of course, be interesting to find global coordinates for $T_{p}$ that do not depend on uniformization by Kleinian groups.

2. Quasifuchsian groups. Let $\Gamma$ be a quasifuchsian group of type $(p, 0)$. This means that the limit set $\Lambda(\Gamma)$ is a Jordan curve in the extended plane, that $\Gamma$ maps each of the Jordan regions $D_{1}$ and $D_{2}$ bounded by $\Lambda(\Gamma)$ into itself, and that the quotient maps $D_{1} \rightarrow D_{1} / \Gamma$ and $D_{2} \rightarrow D_{2} / \Gamma$ are unramified coverings of closed Riemann surfaces of genus $p$.

Lifting a canonical dissection of the surface $D_{1} / \Gamma$ to $D_{1}$, we can choose an ordered $2 p$-tuple

$$
\sigma=\left(A_{1}, B_{1}, A_{2}, B_{2}, \ldots, A_{p}, B_{p}\right)
$$

of Möbius transformations such that the $A_{j}$ and $B_{j}$ generate $\Gamma$ and satisfy the relation

$$
\prod_{j=1}^{p} C_{j}=I, \quad C_{j}=A_{j} B_{j} A_{j}^{-1} B_{j}^{-1}
$$

Received by the editors August 10, 1980; presented to the Society, April 18, 1980.

1980 Mathematics Subject Classification. Primary 32G15; Secondary 30F40.

Key words and phrases. Riemann surface, Teichmüller space, quasifuchsian group.

'This research was partly supported by a grant from the National Science Foundation. 
The pair $(\sigma, \Gamma)$ is called a marked quasifuchsian group. We say that $(\sigma, \Gamma)$ is normalized if the attractive fixed points of $B_{1}$ and $B_{2}$ and the repulsive fixed point of $B_{1}$ are at 0,1 , and $\infty$ respectively. Our reason for that normalization will become clear in $\$ 5$. It is well known (see [3]) that the space of normalized groups $(\sigma, \Gamma)$ is a complex manifold, biholomorphically equivalent to $T_{p} \times T_{p}$.

To represent $T_{p}$ by a set of normalized groups one must embed $T_{p}$ in $T_{p} \times T_{p}$. The Bers coordinates are obtained by identifying $T_{p}$ with a "slice" $T_{p} \times\left\{t_{0}\right\}$ in $T_{p} \times T_{p}$. Our method is to identify $T_{p}$ with the diagonal. Our main theorem gives a general procedure for making that identification, and in $\$ 5$ we illustrate how to use the main theorem to define intrinsic coordinates on $T_{p}$.

3. The main theorem. By definition, if $(\sigma, \Gamma)$ is a marked quasifuchsian group the $2 p$-tuple $\sigma$ induces a canonical dissection of $D_{1} / \Gamma$. The induced dissection of $D_{2} / \Gamma$, however, is not canonical, because of orientation. To identify the space of normalized groups with $T_{p} \times T_{p}$ we use a sense-reversing diffeomorphism to make the dissection of $D_{2} / \Gamma$ canonical. The following theorem describes the diagonal.

THEOREM 1. Let $W$ be a closed Riemann surface of genus $p>2$ with a canonical homotopy basis $a_{1}, \ldots, b_{p}$, and let $\theta$ be an automorphism of $\pi_{1}(W)$ induced by $a$ sense-reversing diffeomorphism of $W$. There is a unique normalized marked quasifuchsian group $(\sigma, \Gamma)$ such that:

(i) the map from $\pi_{1}(W)$ to $\Gamma$ that sends $a_{j}$ to $A_{j}$ and $b_{j}$ to $B_{j}, 1<j<p$, is induced by a conformal map from $W$ to $D_{1} / \Gamma$,

(ii) there is a conformal map $F: D_{2} \rightarrow D_{1}$ such that

$$
F(\gamma z)=\theta(\gamma) F(z) \text { for all } \gamma \in \Gamma, z \in D_{2} .
$$

If $\theta$ has order two, then $F$ is a Möbius transformation of order two, and $F$ and $\Gamma$ generate a Kleinian group whose deformation space is $T_{p}$.

Notice that in (ii) we use the isomorphism (i) between $\pi_{1}(W)$ and $\Gamma$ to interpret $\theta$ as an automorphism of $\Gamma$. We refer the reader to [3] for a discussion of deformation spaces of Kleinian groups.

4. Proof of Theorem 1. This result is really a corollary of the simultaneous uniformization theorem of Bers [1], but we find it simplest to give a direct proof, modelled on the proof of [1]. First we choose a holomorphic universal covering of $W$ by the upper half-plane $U$, identifying $\pi_{1}(W)$ with the group $G$ of deck transformations in the usual way. We normalize $G$ so that the attractive fixed points of $b_{1}$ and $b_{2}$ and the repulsive fixed point of $b_{1}$ are at 0,1 , and $\infty$ respectively. By hypothesis there is a sense-reversing diffeomorphism of $W$ that induces the automorphism $\theta$. Lifting to $U$ we get a diffeomorphism $f: U \rightarrow U$ such that $f(g z)=\theta(g) f(z)$ for all $g \in G, z \in U$. Put $h(z)=f(\bar{z})$ for $z$ in the lower half-plane $U^{*}$. Then $h$ is a sense-preserving diffeomorphism of $U^{*}$ onto $U$, and

$$
h(g z)=\theta(g) h(z) \text { for all } g \in G, z \in U^{*} .
$$

Now let $w: \mathbf{C} \rightarrow \mathbf{C}$ be a quasiconformal map such that $w$ fixes the points 0,1 , and $\infty$, and both $w$ and $w \circ h^{-1}$ are conformal in $U$ (i.e., $w_{\bar{z}}=0$ in $U$ and 
$w_{\bar{z}} / w_{z}=h_{\bar{z}} / h_{z}$ in $\left.U^{*}\right)$. Put $\Gamma=w G w^{-1}$, define the isomorphism $\varphi: G \rightarrow \Gamma$ by

$$
\varphi(g)=w g w^{-1} \text { for all } g \in G,
$$

and put $\sigma=\left(A_{1}, B_{1}, \ldots, A_{p}, B_{p}\right), A_{j}=\varphi\left(a_{j}\right), B_{j}=\varphi\left(b_{j}\right), 1<j<p$. Then $(\sigma, \Gamma)$ is a normalized marked quasifuchsian group with $D_{1}=w(U)$ and $D_{2}=w\left(U^{*}\right)$, and the conformal map $w: U \rightarrow D_{1}$ induces a conformal map of $W$ onto $D_{1} / \Gamma$ that satisfies (i). Moreover $F=w h w^{-1}: D_{2} \rightarrow D_{1}$ is conformal, and (3) and (4) give

$$
F \varphi(g) F^{-1}=w h g h^{-1} w^{-1}=w \theta(g) w^{-1}=\varphi(\theta(g))
$$

in $D_{1}$, so $(\sigma, \Gamma)$ satisfies (i) and (ii).

Suppose $\left(\sigma^{\prime}, \Gamma^{\prime}\right)$ is another normalized group that satisfies (i) and (ii) with $F^{\prime}$ : $D_{2}^{\prime} \rightarrow D_{1}^{\prime}$ conformal. Write $\sigma^{\prime}=\left(A_{1}^{\prime}, \ldots, B_{p}^{\prime}\right)$. Then (i) gives a conformal map $H$ : $D_{1} \rightarrow D_{1}^{\prime}$, so that in $D_{1}^{\prime}$ we have

$$
H A_{j} H^{-1}=A_{j}^{\prime}, \quad H B_{j} H^{-1}=B_{j}^{\prime}, \quad 1<j \leqslant p .
$$

Put $C=H$ in $D_{1}$ and $C=\left(F^{\prime}\right)^{-1} H F$ in $D_{2}$. Then $C$ maps the regular set of $\Gamma$ conformally onto the regular set of $\Gamma^{\prime}$ and induces an isomorphism of $\Gamma$ onto $\Gamma^{\prime}$, so the Marden isomorphism theorem [4] implies that $C$ is a Möbius transformation. The normalization implies that $C$ is the identity, so $(\sigma, \Gamma)=\left(\sigma^{\prime}, \Gamma^{\prime}\right)$ and $(\sigma, \Gamma)$ is unique.

Finally, let $\theta$ have order two. Put $C=F$ in $D_{2}$ and $C=F^{-1}$ in $D_{1}$. Then $C \gamma C^{-1}=\theta(\gamma)$ in both $D_{1}$ and $D_{2}$, so the Marden isomorphism theorem again implies that $C$ is a Möbius transformation. By construction $C$ has order two. Since $F=C$ in $D_{2}, F$ is itself (extendible to) a Möbius transformation of order two. It is clear from (ii) that the group $H$ generated by $\Gamma$ and $F$ is Kleinian, and $\Gamma$ is the subgroup of index two that maps the region $D_{1}$ onto itself. By $\$ 7$ of [3], the deformation space of $H$ is biholomorphically equivalent to $T_{p}$. The equivalence is obtained in the natural way. Each point in the deformation space determines a marked quasifuchsian subgroup $(\sigma, \Gamma)$, which in turn determines a marked Riemann surface $\left(D_{1} / \Gamma, \sigma\right)$ in $T_{p}$. Theorem 1 is proved.

5. Intrinsic global coordinates. To use Theorem 1 we must choose an automorphism $\theta$. Let $(\sigma, \Gamma)$ be a marked quasifuchsian group with $\sigma$ given by (1). Then $\Gamma$ is the free group on $A_{1}, \ldots, B_{p}$, modulo the relation (2). Put

$$
K_{0}=I, \quad K_{j}=\prod_{l=1}^{j} C_{l}, \quad 1<j \leqslant p,
$$

and define $\theta$ on generators by

$$
\theta\left(A_{j}\right)=K_{j-1} A_{j}^{-1} K_{j-1}^{-1}, \quad \theta\left(B_{j}\right)=K_{j} B_{j} K_{j-1}^{-1}, \quad 1<j<p .
$$

It is easy to prove by induction on $j$ that

$$
\theta\left(K_{j}\right)=K_{j}^{-1}, \quad 1<j<p .
$$

The case $j=p$ of (7) shows that $\theta$ preserves the relation (2) and does indeed define an automorphism of $\Gamma$. It is clear from (6) and (7) that $\theta$ has order two. 
Every automorphism of $\Gamma$ is induced by some diffeomorphism of $D_{1} / \Gamma$, and any diffeomorphism that induces $\theta$ is sense-reversing since on the level of homology $\theta$ fixes each $B_{j}$ and reverses each $A_{j}$. We can therefore apply Theorem 1 to $\theta$.

THEOREM 2. If $\theta$ is defined by (6), the normalized group $(\sigma, \Gamma)$ given by Theorem 1 is determined by $B_{1}, \ldots, B_{p}$. The multipliers of the $B_{j}$, the repulsive fixed points of $B_{2}, \ldots, B_{p}$, and the attractive fixed points of $B_{3}, \ldots, B_{p}$ are a global coordinate system for $T_{p}$.

Proof. Let $(\sigma, \Gamma)$ be given by Theorem 1 . Since $\theta$ has order two, there is a Möbius transformation $F$ such that

$$
F^{2}=I \text { and } \theta(\gamma)=F \gamma F^{-1} \text { for all } \gamma \in \Gamma .
$$

Formula (6) implies by induction on $j$ that

$$
\begin{aligned}
K_{j} & =\theta\left(B_{j} \cdots B_{1}\right)\left(B_{j} \cdots B_{1}\right)^{-1} \\
& =F\left(B_{j} \cdots B_{1}\right) F^{-1}\left(B_{j} \cdots B_{1}\right)^{-1}, \quad 1<j<p .
\end{aligned}
$$

Taking $j=p$ in (9) we see that $F$ is the unique Möbius transformation of order two that commutes with the loxodromic transformation $B_{p} \cdots B_{1}$. Thus the $B_{j}$ determine $F$ and, by (9), each $K_{j}$.

Now put

$$
F_{j}=F K_{j-1} A_{j}, \quad 1<j<p .
$$

We claim that $F_{j}$ is the unique Möbius transformation of order two that commutes with $B_{j}$. First, $F_{j}$ is not the identity map since it interchanges the regions $D_{1}$ and $D_{2}$. Next,

$$
F_{j}^{2}=F K_{j-1} A_{j} F K_{j-1} A_{j}=\theta\left(K_{j-1} A_{j}\right) K_{j-1} A_{j}=I,
$$

by (6), (7), and (8). Finally,

$$
\begin{aligned}
F_{j} B_{j} F_{j}^{-1} & =F_{j} B_{j} F_{j}=\theta\left(K_{j-1} A_{j} B_{j}\right) K_{j-1} A_{j} \\
& =A_{j}^{-1} K_{j-1}^{-1} K_{j} B_{j} A_{j}=A_{j}^{-1} C_{j} B_{j} A_{j}=B_{j},
\end{aligned}
$$

which proves our claim.

Since the $B_{j}$ uniquely determine $F$ and the $F_{j}$, formulas (9) and (10) show that the $B_{j}$ determine $(\sigma, \Gamma)$. That proves the first statement of Theorem 2 . The second statement follows easily. Indeed, the fixed points and multipliers of the $B_{j}$ are holomorphic functions on the deformation space of the Kleinian group generated by $\Gamma$ and $F$ (see $\S 8$ of [3]), hence on $T_{p}$. Since these fixed points and multipliers determine the $B_{j}$, and hence $(\sigma, \Gamma)$, we have defined an injective holomorphic map from $T_{p}$ into $C^{n}$. The theorem is proved.

We remark in conclusion that $\left\{B_{1}, \ldots, B_{p}\right\}$ generates a Schottky group of genus $p$, and our coordinates on $T_{p}$ give an injective holomorphic map of $T_{p}$ into the space of Schottky groups of genus $p$. We will study the geometry of that map in a forthcoming paper. 


\section{REFERENCES}

1. L. Bers, Simultaneous uniformization, Bull. Amer. Math. Soc. 66 (1960), 94-97.

2. __ Correction to "Spaces of Riemann surfaces as bounded domains", Bull. Amer. Math. Soc. 67 (1961), 465-466.

3. I. Kra, Deformation spaces, 'A crash course on Kleinian groups', Lecture Notes in Math., vol. 400 , Springer-Verlag, Berlin and New York, 1974, pp. 48-70.

4. A. Marden, Geometrically finite Kleinian groups and their deformation spaces, 'Discrete Groups and Automorphic Functions', Academic Press, London and New York, 1977, pp. 259-293.

5. B. Maskit, Moduli of marked Riemann surfaces, Bull. Amer. Math. Soc. 80 (1974), 773-777.

Department of Mathematics, Cornell University, Ithaca, New York 14853 